\title{
Using particle swarm optimization to solve test functions problems
}

\author{
Issa Ahmed Abed, May Mohammed Ali, Afrah Abood Abdul Kadhim \\ Basrah Engineering Technical College, Southern Technical University, Iraq
}

\begin{tabular}{l} 
Article Info \\
\hline Article history: \\
Received May 22, 2021 \\
Revised Aug 17, 2021 \\
Accepted Oct 6, 2021 \\
\hline
\end{tabular}

Keywords:

Best solution

Comparison

Operators

Optimization

Warm

\begin{abstract}
In this paper the benchmarking functions are used to evaluate and check the particle swarm optimization (PSO) algorithm. However, the functions utilized have two dimension but they selected with different difficulty and with different models. In order to prove capability of PSO, it is compared with genetic algorithm (GA). Hence, the two algorithms are compared in terms of objective functions and the standard deviation. Different runs have been taken to get convincing results and the parameters are chosen properly where the Matlab software is used. Where the suggested algorithm can solve different engineering problems with different dimension and outperform the others in term of accuracy and speed of convergence.
\end{abstract}

This is an open access article under the CC BY-SA license.

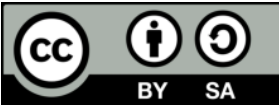

\section{Corresponding Author:}

Issa Ahmed Abed

Department of Automation and Control Technologies Engineering

Basrah Engineering Technical College

Southern Technical University, Basrah, Iraq

Email: issaahmedabed@ stu.edu.iq

\section{INTRODUCTION}

A large number of real-life optimization problems in science, engineering, economics, and business are complex and difficult to solve. They cannot be solved in an exact manner within a reasonable amount of time. Using approximate algorithms is the main alternative to solve this class of problems [1], [2]. The optimization process involves finding a single or a series of optimal solutions from among a very large number of possibilities. In this process, the space of potential solutions is reduced to one or a few of the best ones [3], [4]. Many researchers constructed different methods to get the best solution because it is difficult to find the solution in many problems in engineering. Particle swarm optimization (PSO) is stochastic method depends on behavior of animals. Wang et al. [5] gave the basic and the details of PSO. They analyzed the method from various views of that are the structure, parameters, discrete PSO, parallel PSO, and multiobjective. Ozdemir [6] used the PSO to reach to the global minimum of the function suggested. He applied the method on different benchmark functions. The author proved that PSO is a successful algorithm to solve various problems. Jumaa et al. [7] suggested a method for scheduling optimization and running of distributed generator to reduce the loss of power, revamp voltage profile as well as the reliability of the whole network. They proposed particle swarm optimization in order to estimate the better site and the size of distributed generation. Garcia et al. [8] estimated a technique utilizing genetic algorithm (GA) to evaluate the relation maximum of differentiable functions. The authors introduced Python library contains components from the algorithm. Alyoutbaki and Al-Rawi [9] introduced a method to extend the fault tolerance of the system. Ant colony optimization (ACO) is used to enhance the suggested strategy. ACO can select better virtual machine where is to emigrate the cloudlet in order to decrease the consumption of energy and the time. 
This paper presents the particle swarm optimization to solve some suggested test functions. The rest of the paper is organized as; section 2 is the overview of genetic algorithm and PSO; comparison between the both algorithms is introduced in section 3; the simulation results are in section 4; section 5 is the conclusion.

\section{THE PROPOSED METHODS}

\subsection{Genetic algorithm}

In order to solve many optimization problems, genetic algorithm is used which is considered as an adaptive technique. According to the selection of natural and the survival of fittest the population will promote through different iterations. If the genetic algorithm has been encoded properly in that case it is able to transfer the solution from simulation to the real applications where the GA will simulate the selection and survival [10], [11]. In this algorithm, the size of population of individuals is fixed constant, where the GA goes generation after generation. During the iterations of GA, it will pass through various operations such as reproduction, crossover, and mutation which produce new individuals offspring. The new population is rated by the function which is called objective function. The best new solutions are calculated by these procedures [12]-[14].

\subsection{Particle swarm optimization}

Particle swarm optimization is first given by Erberhart and Kennedy. It is an individual's population which are called particles. PSO is inspired from the behavior of flocking bird and the schooling fish [15], [16]. It is a technique depends on the relation between the particles of swarm where it is an optimization stochastic and the population intellects in the search space. Here, there is position and velocity for each particle. These values are changed through the generations where the best position is the particles of best experience and the global position is the best achieved experience of all particles [17], [18]. Also, the particles have vectors of numbers which are real and every vector position is called dimension. Two relations are there, first for velocity and the second for position which are illustrated in (1) and (2) [19], [20]:

$$
\begin{gathered}
\operatorname{vel}_{i, j}^{g e n+1}=\text { weight } * \operatorname{vel}_{i, j}^{g e n}+\operatorname{acc}_{1} * \operatorname{rand}_{1} *\left(\text { perbest }_{i, j}^{g e n}-\text { par }_{i, j}^{g e n}\right)+\operatorname{acc}_{2} \\
* \operatorname{rand}_{2} *\left(\text { globalbest }_{i, j}^{g e n}-\operatorname{par}_{i, j}^{g e n}\right) \\
\operatorname{par}_{i, j}^{g e n+1}=\operatorname{par}_{i, j}^{g e n}+\operatorname{vel}_{i, j}^{g e n+1}
\end{gathered}
$$

Where $\operatorname{par}_{i}$ represents the particles in the population of size ( $\left.p o p\right)$ with the dimension of (dim) and $\operatorname{par}_{i}=\left\{\operatorname{par}_{i, 1}, \operatorname{par}_{i, 2}, \ldots \ldots \ldots, \operatorname{par}_{i, d i m}\right\}$. In addition, the population has velocity $(v e l)$ and it is written as, $\operatorname{vel}_{i}=\left\{\operatorname{vel}_{i, 1}, \operatorname{vel}_{i, 2}, \ldots \ldots \ldots\right.$, vel $\left._{i, \mathrm{dim}}\right\}$. The $(i)$ is from 1 to $(\mathrm{pop}),(j)$ from 1 to $(\mathrm{dim})$ and $(\mathrm{gen})$ is the iteration number. The (perbest $\left.i_{i, j}^{\text {gen }}\right)$ is the personal best with $\left(j^{\text {th }}\right)$ parameter of $\left(i^{\text {th }}\right)$ individual and the

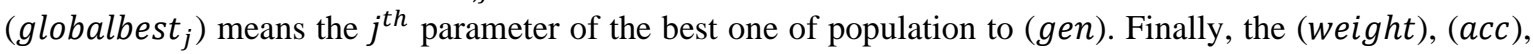
and (rand) are the weight parameter, acceleration parameter and the random number between [0,1] [21][23]. Figure 1 shows the overall algorithm.

\subsection{The difference between PSO and some other algorithms}

The difference between PSO and some other algorithms. The following steps are found in many evolutionary methods:

- In the initialization there is a random individual.

- The range to reach to the optimum will be the base for the objective function which is calculated for each part.

- The objective value is also the base for the reproduction of population.

- These procedures can stop or continue.

In that case the PSO has the same points like many algorithms. Therefore, they are generated the population randomly. They are depended on the objective function to calculate the best solution. All of them goes from step to step to reach to the best, but they do not pledge the winning. Hence, the PSO for example does not contains processes like crossover or mutation. Where it is enhancing their particles by the internal velocity and PSO contains memory. However, there are some differences between PSO and other algorithms such as GA. Where all the individuals in GA translate the information between them while the in the PSO only globalbest provides the information to the other individuals [24]. 


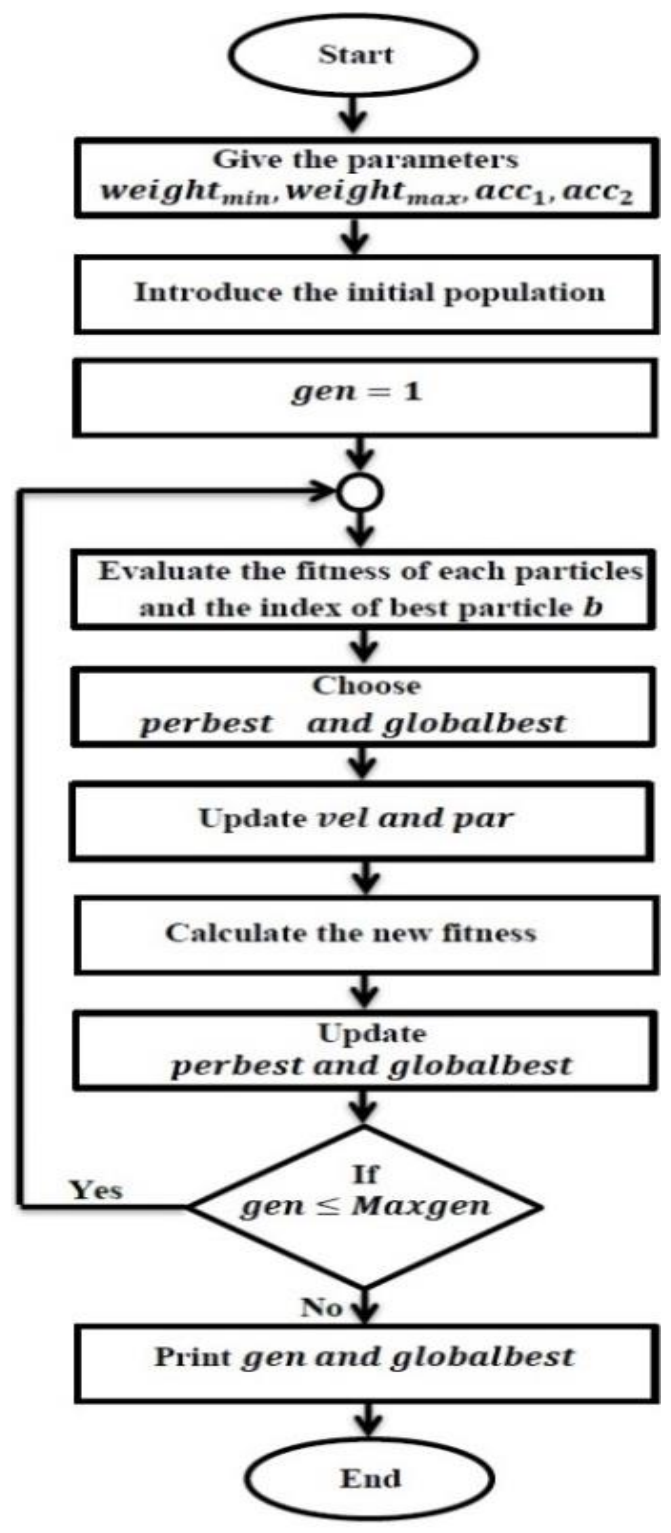

Figure 1. PSO procedures

\section{RESULTS AND DISCUSSION}

The tests have been done on the PC Intel Core i7 processor $2.7 \mathrm{GHz}$ and windows 7 professional with 4 GB RAM. All the results are simulated using Matlab 2008a. The population size was 10 while the number of iterations are equal to 100. In addition, $p_{m}=0.5$ in GA and in PSO the inertia weight between 0.4 and 0.9 while the acceleration coefficients are 2 . In order to test the ability of PSO, the following functions have been used in this paper where all of them are two dimensional [25].

\subsection{Beale function}

This is the first function suggested here. The mathematical formula is:

$$
f(x)=\left(1.5-x_{1}+x_{1} x_{2}\right)^{2}+\left(2.25-x_{1}+x_{1} x_{2}^{2}\right)^{2}+\left(2.625-x_{1}+x_{1} x_{2}^{3}\right)^{2}
$$

Which is a multimodal function. The $x_{1,2} \in[-4.5,4.5]$ with $f\left(x^{\text {best }}\right)=0$ at $x^{\text {best }}=(3,0.5)$. The details of this function are shown in Figure 2. The particle swarm optimization has been tested through the Beale function and it is compared with genetic algorithm as illustrated in Figure 3. It is obvious that the PSO is faster that GA as well it is more accurate to use in this function. 


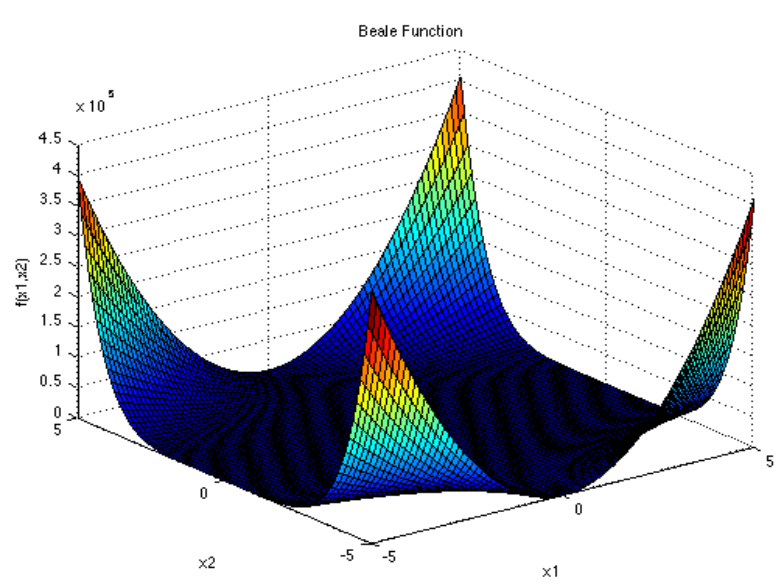

Figure 2. The image of Beale function

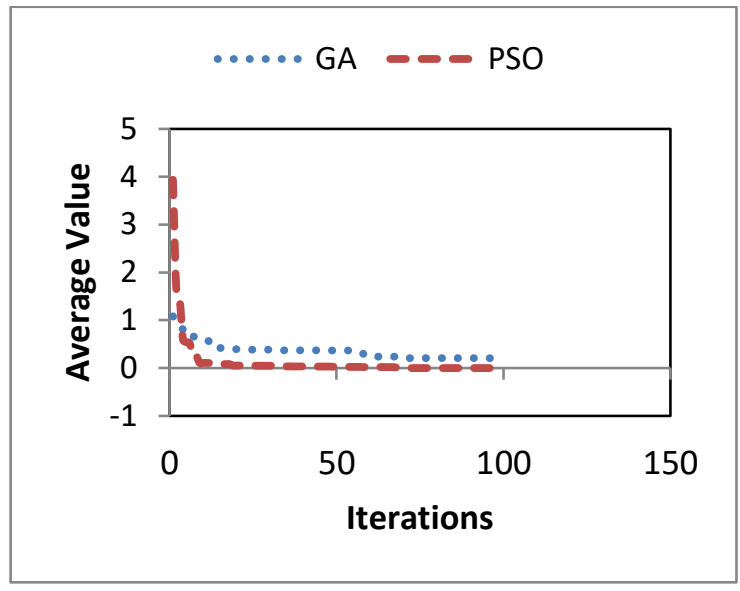

Figure 3. Functions comparison

\subsection{McCormick function}

The common relation for this function is:

$$
f(x)=\sin \left(x_{1}+x_{2}\right)+\left(x_{1}-x_{2}\right)^{2}-1.5 x_{1}+2.5 x_{2}+1
$$

The boundary of dimensions $x_{1} \in[-1.5,4], x_{2} \in[-3,4]$ while the optimum $f\left(x^{\text {best }}\right) \approx$ -1.9133 at $x^{\text {best }}=(-0.547,-1.547)$. Figure 4 presents this function. Also, the algorithms are tested through McCormick function to show the ability of each method as shown in Figure 5. From the figure it can be seen, there is a big comparable between the methods but still the PSO outperforms the GA and can reach the best solution.

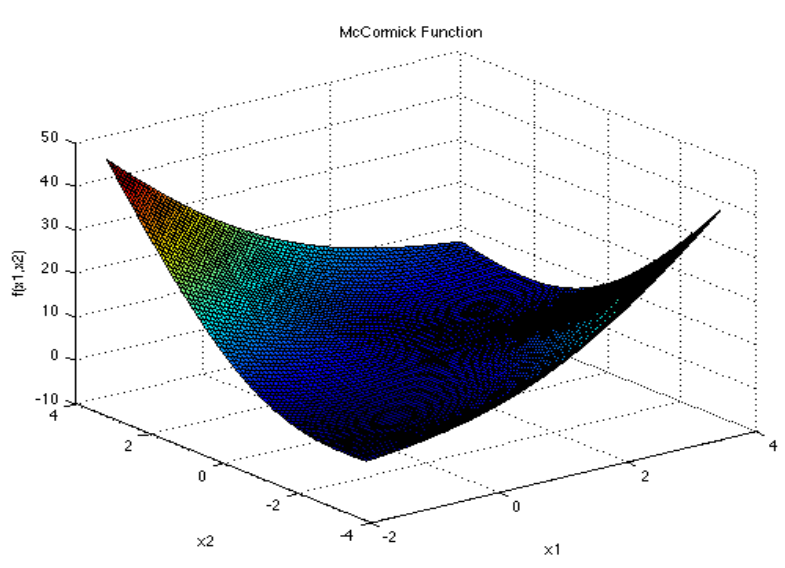

Figure 4. The image of McCormick function

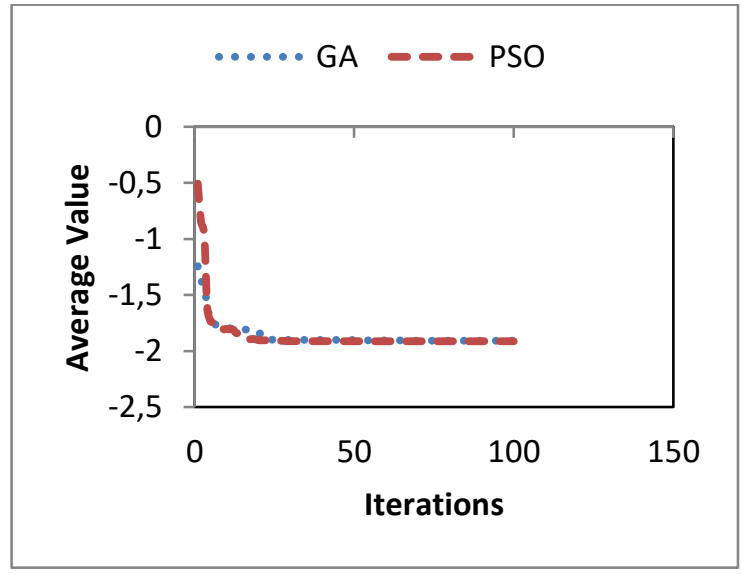

Figure 5. The PSO comparison

\subsection{Matyas function}

The expression for this function is:

$$
f(x)=0.26\left(x_{1}^{2}+x_{2}^{2}\right)-0.48 x_{1} x_{2}
$$

Where the parameters $x_{1,2} \in[-10,10]$ and the best function $f\left(x^{\text {best }}\right)=0$ at $x^{\text {best }}=(0,0)$. The graph of Matyas function is given in Figure 6. From Figure 7 which shows the value for the two methods during the generations. The value by using the PSO converges generation by generation to reach to the target point in very suitable time. 


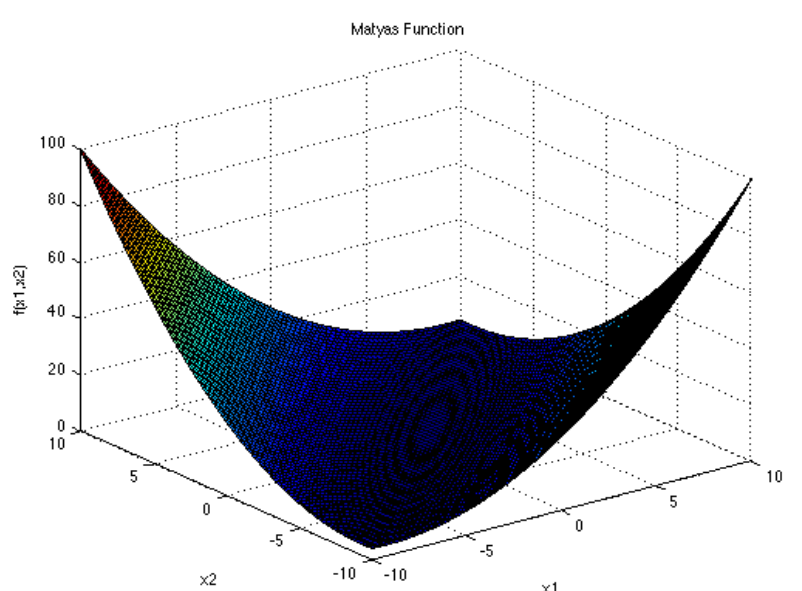

Figure 6. The 3-D graph for matyas

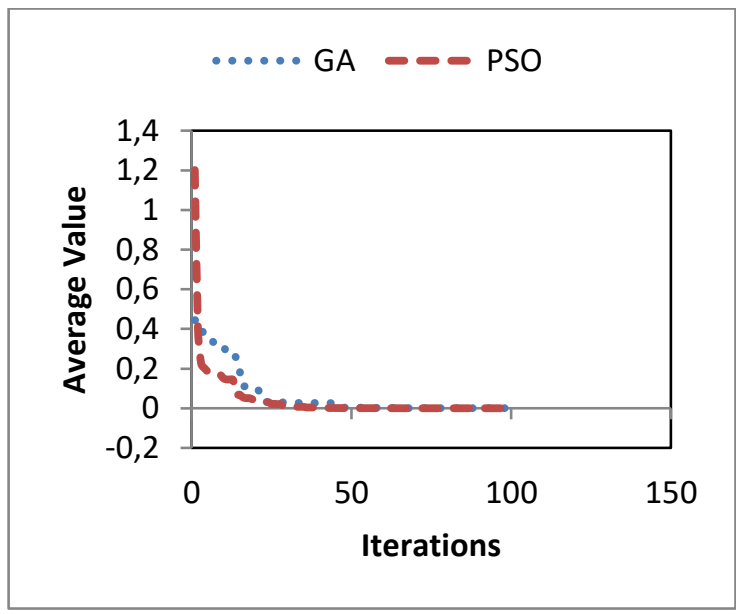

Figure 7. The convergence of the methods

\subsection{Mishra bird function}

It is a function with formula:

$$
f(x)=\sin \left(x_{1}\right) e^{\left(1-\cos \left(x_{2}\right)\right)^{2}}+\cos \left(x_{2}\right) e^{\left(1-\sin \left(x_{1}\right)\right)^{2}}+\left(x_{1}-x_{2}\right)^{2}
$$

The dimensions should be $x_{1,2} \in[-2 \pi, 2 \pi]$ while the fuction is $f\left(x^{\text {best }}\right)=-106.764537$ at $\boldsymbol{x}^{\text {best }}=(4.70104,3.15294)$ and $x^{\text {best }}=(-1.58214,-3.13024)$. The graph for this function is given in Figure 8 . The PSO still has the best position in terms of the error and the speed and the GA cannot overcome this algorithm as presented in Figure 9.

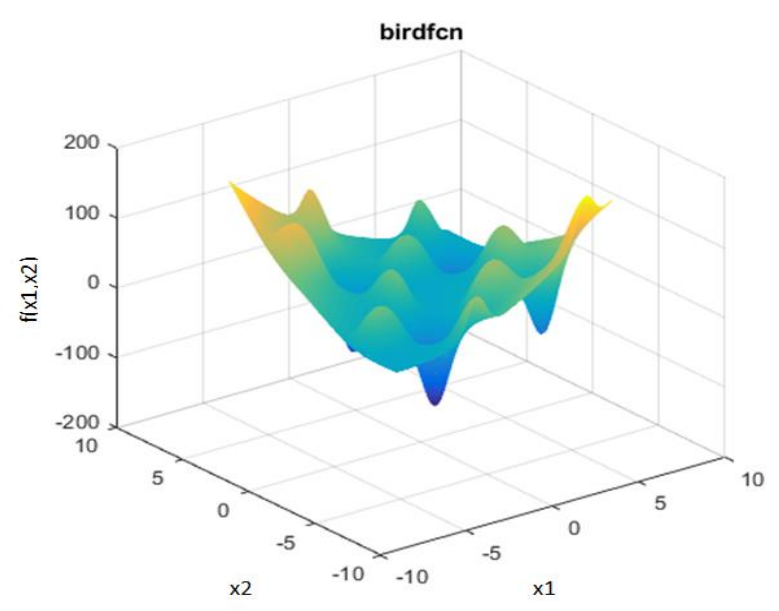

Figure 8 . The function of mishra

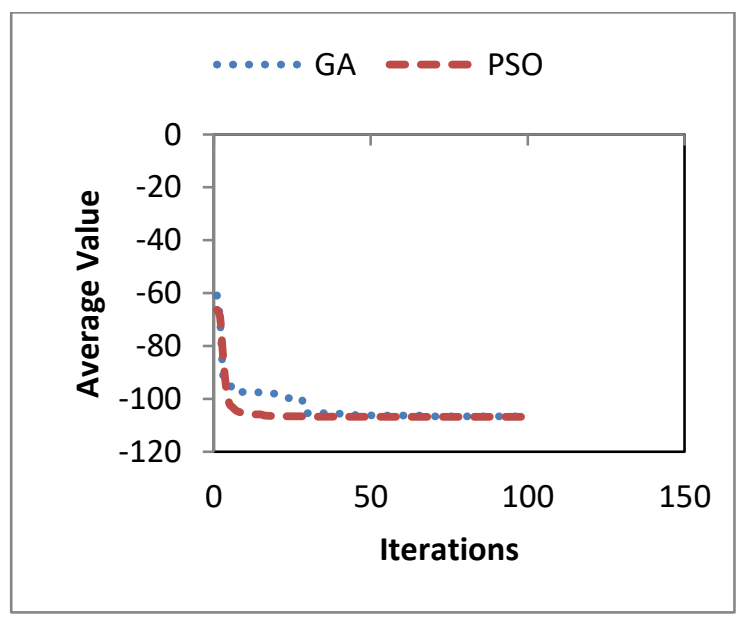

Figure 9. The comparison curves between the two in Mishra bird

\subsection{Holder table function}

The mathematical equation for this function is:

$$
f(x)=-\left|\sin \left(x_{1}\right) \cos \left(x_{2}\right) \exp \left(\left|1-\frac{\sqrt{x_{1}^{2}+x_{2}^{2}}}{\pi}\right|\right)\right|
$$


In that case $x_{1,2} \in[-10,10]$ and $f\left(x^{\text {best }}\right)=-19.2085$ at $x^{\text {best }}=(8.05502,9.66459)$, $(-8.05502,9.66459),(8.05502,-9.66459),(-8.05502,-9.66459)$. The graph of holder function is illustrated in Figure 10. In this test which seems to be difficult for PSO which is trapped in local optima where the accuracy is less than the other algorithm as shown in Figure 11.

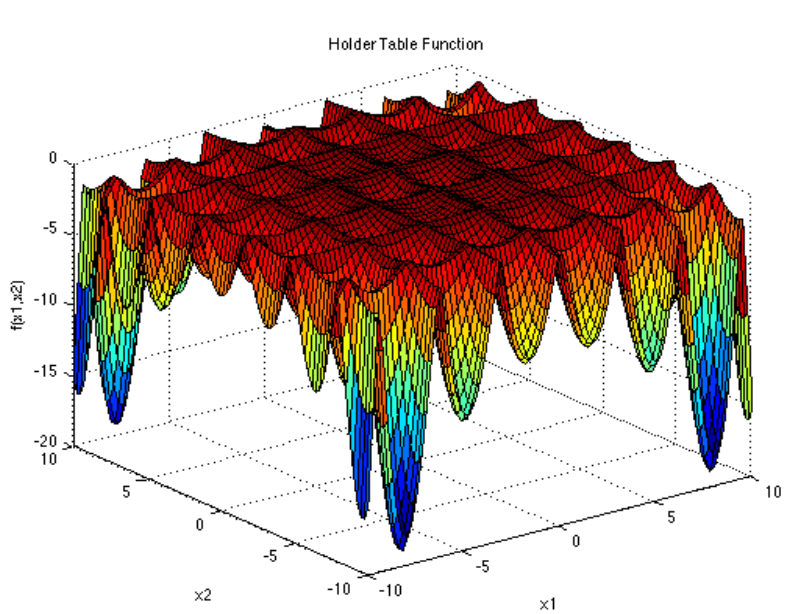

Figure 10. The function of holder table

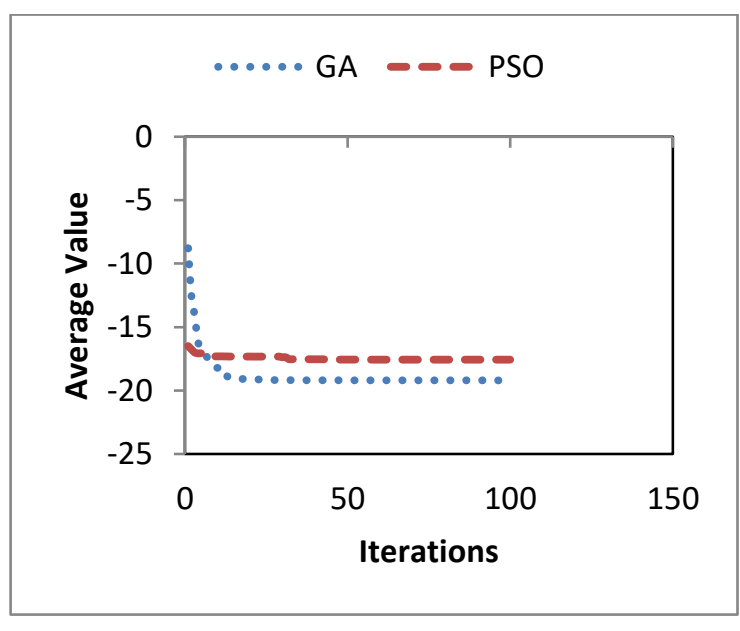

Figure 11. The comparison between the two algorithms

\subsection{Eggholder function}

The equation is:

$$
f(x)=-\left(x_{2}+47\right) \sin \left(\sqrt{\left|x_{2}+\frac{x_{1}}{2}+47\right|}\right)-x_{1} \sin \left(\sqrt{\left|x_{1}-\left(x_{2}+47\right)\right|}\right.
$$

At $x_{1,2} \in[-512,512]$ with $f\left(x^{\text {best }}\right)=-959.6407$ for $x^{\text {best }}=(512,404.2319)$ and the graph for this function is introduced in Figure 12. Figure 13 shown the GA is still dominant on GA and they are very comparative in terms of the speed.

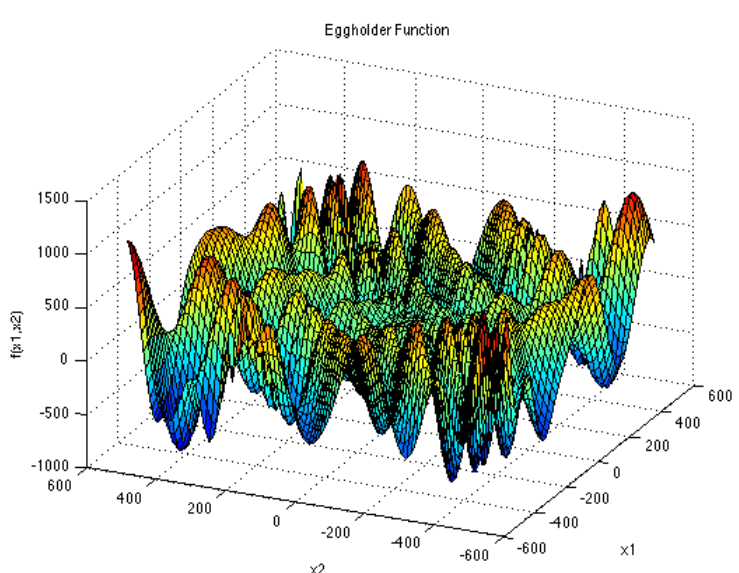

Figure 12. The graph Egg function

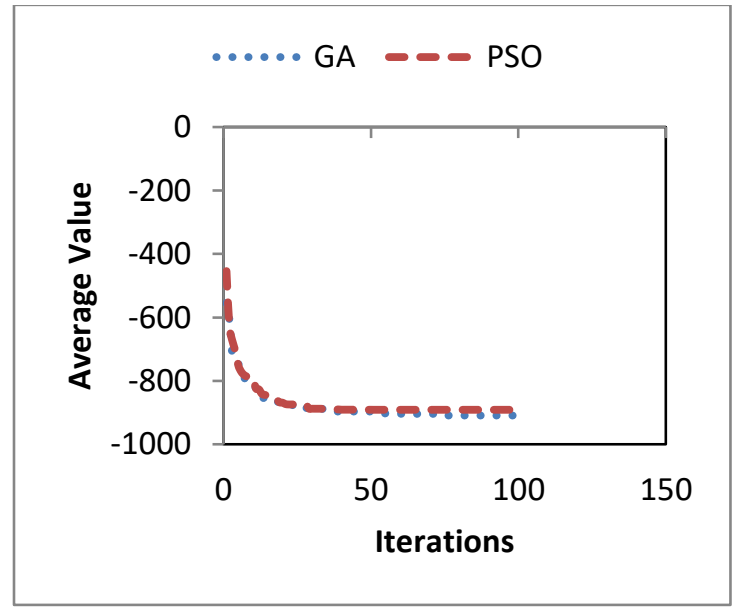

Figure 13. The objective curves

\subsection{Schaffer function}

The Mathematical equation is: 


$$
f(x)=0.5+\frac{\sin ^{2}\left(x_{1}^{2}-x_{2}^{2}\right)-0.5}{\left[1+0.001\left(x_{1}^{2}+x_{2}^{2}\right)\right]^{2}}
$$

Where $x_{1,2} \in[-100,100]$ and $f\left(x^{\text {best }}\right)=0$, with $x^{\text {best }}=(0,0)$ as shown in Figure 14. In this function, even if PSO is better, but both algorithms need more improvement to approximate to the optimum value which is 0 as shown in Figure 15.

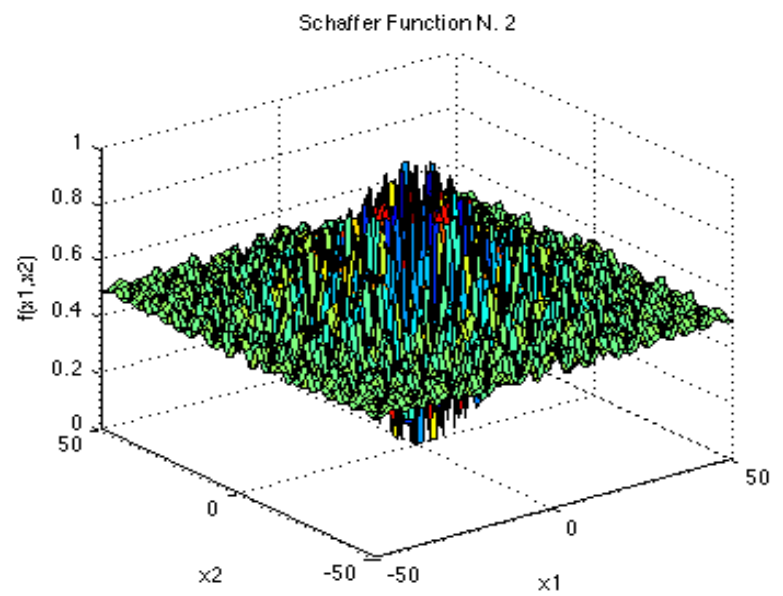

Figure 14. The function of Schaffer

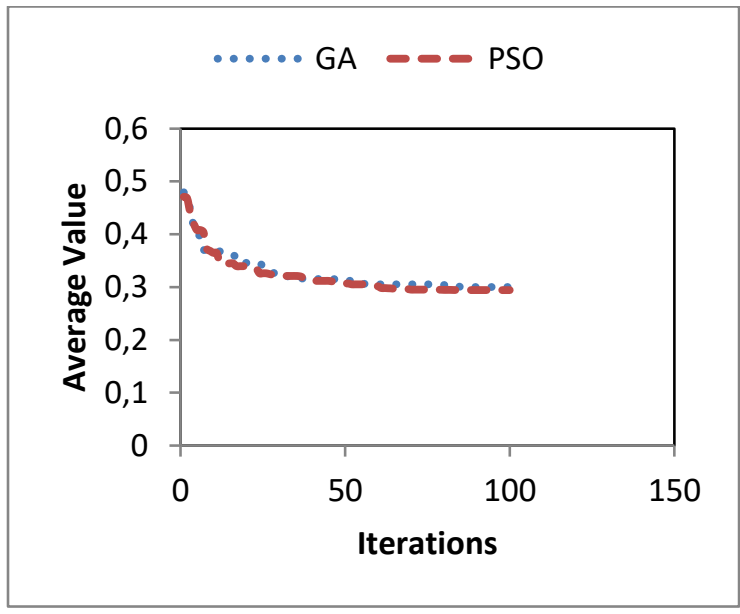

Figure 15. The approximation of the algorithms

\subsection{Booth function}

This function is:

$$
f(x)=\left(x_{1}+2 x_{2}-7\right)^{2}+\left(2 x_{1}+x_{2}-5\right)^{2}
$$

The dimensions $x_{1,2} \in[-10,10]$ and $f\left(x^{\text {best }}\right)=0$ at $x^{\text {best }}=(1,3)$ as shown in Figure 16. In this function the PSO won to reach faster and gets the optimal solution as given in Figure 17.

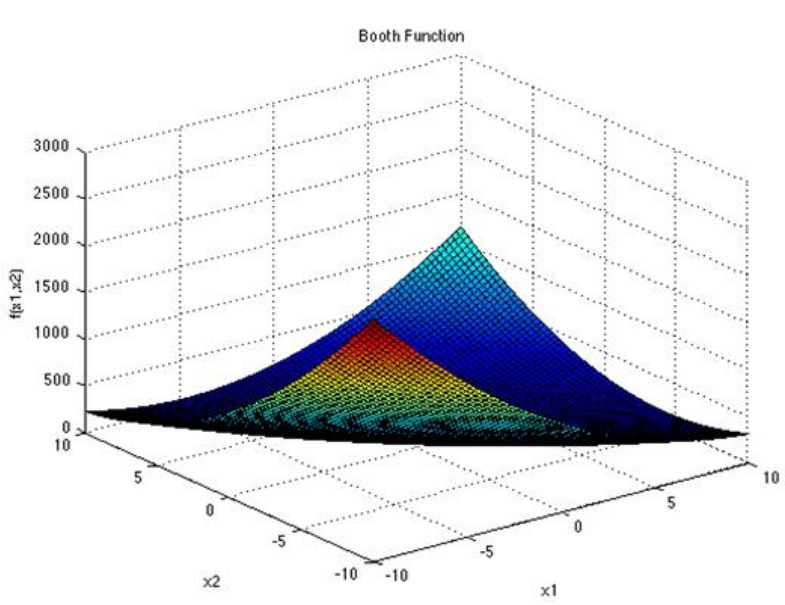

Figure 16. Booth image

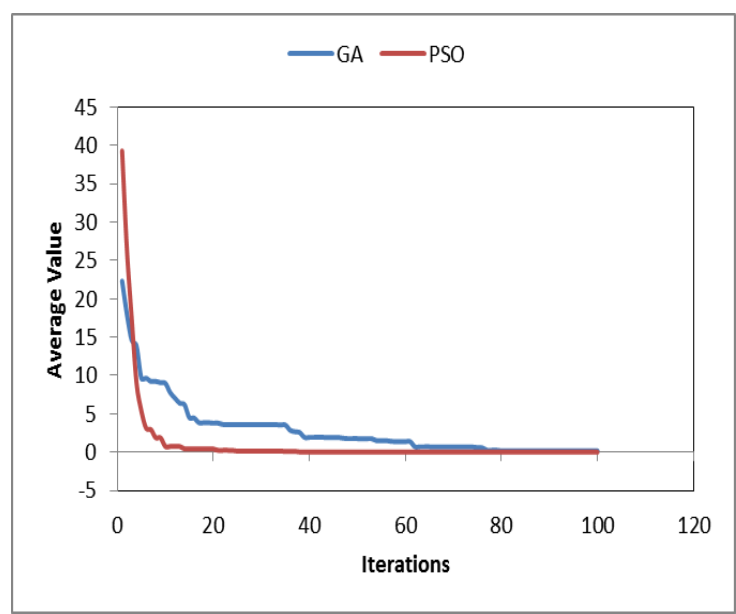

Figure 17. Function approximation

\subsection{Easom function}

The mathematical expression for this function is: 


$$
f(x)=-\cos \left(x_{1}\right) \cos \left(x_{2}\right) \exp \left(-\left(x_{1}-\pi\right)^{2}-\left(x_{2}-\pi\right)^{2}\right)
$$

It is a unimodal with $x_{1,2} \in[-100,100]$ with $f\left(x^{\text {best }}\right)=-1$, at $x^{\text {best }}=(\pi, \pi)$ as shown in Figure 18. In this function, the GA do very bad while the PSO is excellent and it is reached the target solution in better way as presented in Figure 19.

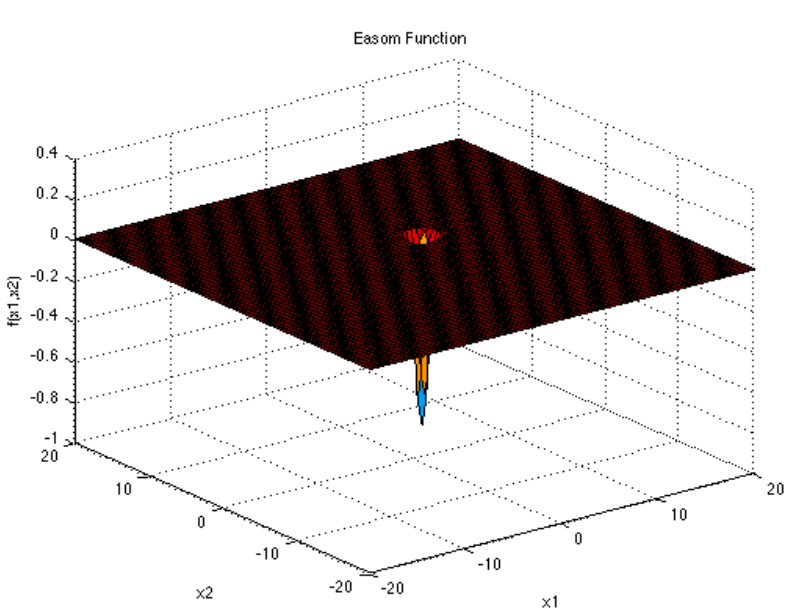

Figure 18. The easom function

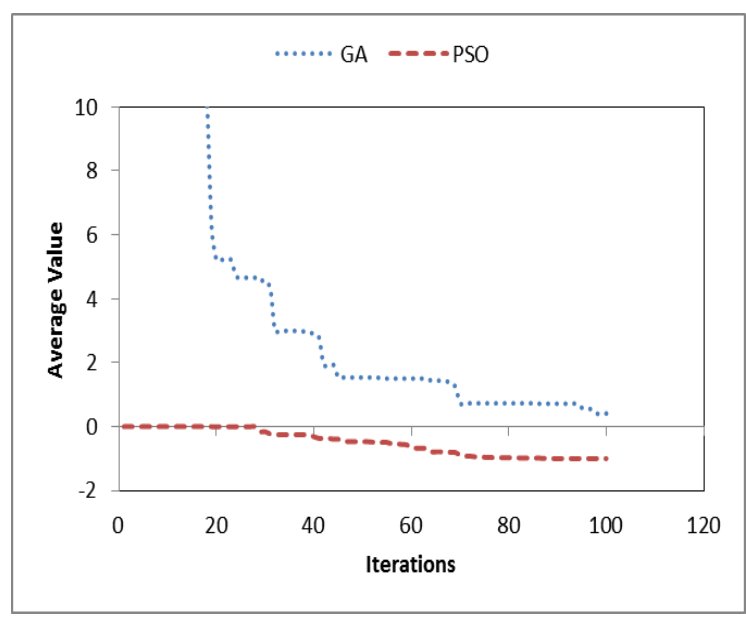

Figure 19. Comparision function

Table 1 is the summary for average value and the deviation for both the algorithms with different suggested test functions where it is clearly that the PSO is dominant. Additional results are given in Table 2 for one check during the work.

Table 1. The SD and the average for different functions

\begin{tabular}{ccccc}
\hline \multirow{2}{*}{ Function } & \multicolumn{2}{c}{ Particle swarm optimization } & \multicolumn{2}{c}{ Genetic algorithm } \\
\cline { 2 - 5 } & Standard deviation & Average & Standard deviation & Average \\
\hline Beale & 0 & 0 & 0.330022 & 0.20556 \\
McCormick & $2.48 \mathrm{E}-16$ & -1.9132 & 0.005451 & -1.9086 \\
Matyas & 0 & 0 & 0.000572 & 0.00058 \\
Mishra bird & 0 & -106.788 & 0.158935 & -106.614 \\
Holder table & 1.814672 & -17.5691 & 0 & -19.2014 \\
Eggholder & 69.2471 & -891.251 & 74.95725 & -909.32 \\
Schaffer & 0.003303 & 0.29434 & 0 & 0.3002 \\
Booth & 0 & 0 & 0.214113 & 0.18532 \\
Easom & $5.48 \mathrm{E}-5$ & -1 & 0.740961 & 0.40972 \\
\hline
\end{tabular}

Table 2. Different results for suggested problems

\begin{tabular}{ccccc}
\hline \multirow{2}{*}{ Function } & \multicolumn{2}{c}{ Particle swarm optimization } & \multicolumn{2}{c}{ Genetic algorithm } \\
\cline { 2 - 5 } & Best value & Best variables & Best value & Best variable \\
\hline Beale & $1.45 \mathrm{e}-008$ & $3.0002,0.5$ & 0.2303 & $2.2412,0.2647$ \\
McCormick & -1.9132 & $-0.5472,-1.5472$ & -1.9121 & $-0.5294,-1.5176$ \\
Matyas & $7.229 \mathrm{e}-012$ & $-0.1353,-0.1163$ & $6.1515 \mathrm{e}-005$ & $0.0392,0.0392$ \\
Mishra bird & -106.7877 & $-3.1229,-1.5895$ & -106.7139 & $3.0980,-1.6059$ \\
Holder table & -19.2085 & $8.055,9.6646$ & -19.2014 & $8.0392,9.6863$ \\
Eggholder & -959.6407 & $512.00,404.2318$ & -950.5854 & $475.8588,431.6863$ \\
Schaffer & 0.2923 & $1.2651,0.1700$ & 0.3002 & $-1.1765,-3.5294$ \\
Booth & $3.0702 \mathrm{e}-010$ & $1.00,3.00$ & 0.1576 & $1.2941,2.7843$ \\
Easom & -1.00 & $3.1417,3.1416$ & -1.2162 & $-0.3922,-0.3922$ \\
\hline
\end{tabular}

\section{CONCLUSION}

Particle swarm optimization test has been done to highlight the ability of the algorithm to optimize the difficult problems. The experiments have included various mathematical functions with details presents in this paper. In addition to that, it is compared with other algorithm to be sure that this algorithm can 
outperform the others in terms of the accuracy and the speed. Hence, this method can be used to solve any optimization problem in all the fields.

\section{ACKNOWLEDGEMENTS}

Many thanks for everybody in Southern Technical University how help us to complete this paper.

\section{REFERENCES}

[1] T. El-Ghazali, "Metaheuristics: from design to implementation," vol. 74, John Wiley \& Sons, 2009

[2] H. W. Abdulrazak, I. A. Abed, and D. K. Shary. "Management of Microgrid System Based on Optimization Algorithm," Journal of Physics: Conference Series, vol. 1773. no. 1, 2021.

[3] Z. Jozef, "Optimization Problems and Genetic Algorithms," Review of Business Information Systems, vol. 14, no. 3 2010, doi: 10.19030/rbis.v14i3.491.

[4] G. M. Fadhil, I. A. Abed and R. S. Jasim, "Genetic Algorithm Utilization to Fine Tune the Parameters of PID Controller," Kufa Journal of Engineering, vol. 12, no. 2, pp. 1-12, 2021, doi: 10.30572/2018/KJE/120201.

[5] D. Wang, D. Tan and L. Lie, "Particle swarm optimization algorithm: an overview," Soft Computing, vol. 22, pp. 387-408, 2018.

[6] M. Ozdemir, "Particle swarm optimization for continuous function optimization problems," International Journal of Applied Mathematics Electronics and Computers, vol. 5, no. 3, pp. 47-52, 2017, doi: 10.18100/ijamec.2017331879.

[7] F. A. Jumaa, O. M. Neda, and M. A. Mhawesh, "Optimal distributed generation placement using artificial intelligence for improving active radial distribution system," Bulletin of Electrical Engineering and Informatics, vol. 10, no. 5, pp. 2345-2354, doi: 10.11591/eei.v10i5.2949.

[8] J. M. Garcia, C. A. Acosta and M. J. Mesa, "Genetic algorithms for mathematical optimization," Journal of Physics: Conference Series, vol. 1448, no. 1, 2020.

[9] Y. A. G. Alyouzbaki and M. F. Al-Rawi, "Novel load balancing approach based on ant colony optimization technique in cloud computing," Bulletin of Electrical Engineering and Informatics, vol. 10, no. 4, pp. 2320-2326, 2021, doi: 10.11591/eei.v10i4.2947.

[10] M. S. Sami, and H. A. Kubba, "Genetic algorithm based load flow solution problem in electrical power systems," Journal of Engineering, vol. 15 no. 4, pp. 4142-4162, 2009.

[11] B. Tarek, L. Slimani, and M. Belkacemi, "A genetic algorithm for solving the optimal power flow problem," Leonardo Journal of Sciences, vol. 4, pp. 44-58, 2004.

[12] Younes, Mimoun, and Mostefa Rahli, "On the choice genetic parameters with Taguchi method applied in economic power dispatch," Leonardo journal of sciences, vol. 9, pp. 9-24, 2006.

[13] D. Hermawanto, "Genetic algorithm for solving simple mathematical equality problem," arXiv preprint arXiv:1308.4675, 2013.

[14] ABM. Nasiruzzaman, and MG. Rabbani, "A Genetic Algorithm Based Approach for Solving Optimal Power Flow Problem," International Conference on Electronics, Computer and Communication, 2008.

[15] J. Kennedy and R. Eberhart, "Particle swarm optimization," Proceedings of ICNN'95 - International Conference on Neural Networks, 1995, vol. 4, pp. 1942-1948, doi: 10.1109/ICNN.1995.488968.

[16] E. A. Kaur and E. K. Mandeep, "A comprehensive survey of test functions for evaluating the performance of particle swarm optimization algorithm," International Journal of Hybrid Information Technology, vol. 8, no. 5, pp. 97-104, 2015.

[17] M. Khesti and L. Ding, "Particle Swarm Optimization Solution for Power System Operation Problems," Particle Swarm Optimization with Applications. IntechOpen, 2017.

[18] S. M. Majercik, "GREEN-PSO: Conserving Function Evaluations in Particle Swarm Optimization," IJCCI, 2013.

[19] G. Tomasseti and L. Cagnina, "Particle swarm algorithms to solve engineering problems: a comparison of performance," Journal of Engineering, vol. 2013, no. 435104, 2013, doi: 10.1155/2013/435104

[20] M. R. Bonyadi and Z. Michalewicz, "Particle Swarm Optimization for Single Objective Continuous Space Problems: A Review," Evolutionary Computation, vol. 25, no. 1, pp. 1-54, 2017, doi: 10.1162/EVCO_r_00180.

[21] MN. Alam, "Particle swarm optimization: Algorithm and its codes in matlab," ResearchGate, pp. 1-10, 2016.

[22] P. Erdoğmus, "Particle swarm optimization performance on special linear programming problems," Scientific Research and Essays, vol. 5, no. 12, pp. 1506-1518, 2013.

[23] BSG. D. Almeida and VC. Leite, "Particle Swarm Optimization: A Powerful Technique for Solving Engineering Problems," Swarm Intelligence-Recent Advances, New Perspectives and Applications, IntechOpen, 2019.

[24] M. Najjarzadeh and A. Ayatollahi, "A comparison between Genetic Algorithm and PSO for linear phase FIR digital filter design," 9th International Conference on Signal Processing, 2008, pp. 2134-2137, doi: 10.1109/ICOSP.2008.4697568.

[25] I. A. Abed, S. P. Koh, K. S. M. Sahari, S. K. Tiong, M. M. Ali and A. A. A. Kadhim, "A New Proposed PendulumLike with Attraction-Repulsion mechanism Algorithm for Solving Optimization Problems," IOP Conference Series: Materials Science and Engineering, vol. 881, no. 1, p. 012132, 2020. 


\section{BIOGRAPHIES OF AUTHORS}
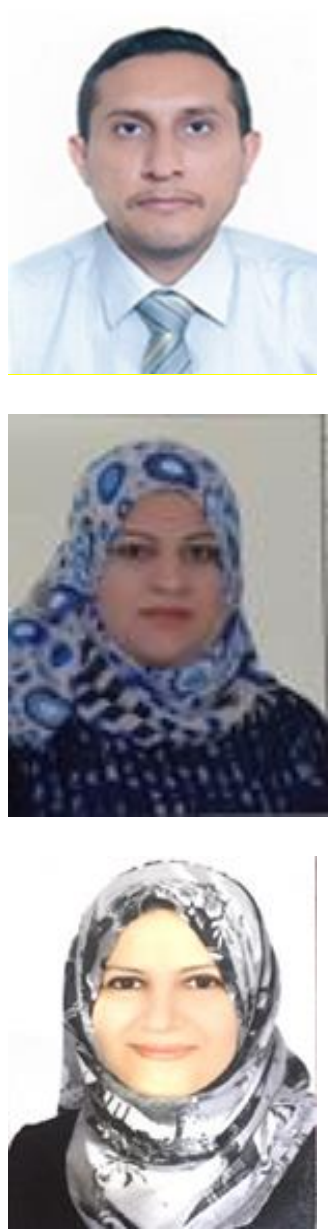

May Mohammed Ali received the B.Sc. and M.Sc. degrees in electrical engineering from AlBasrah University, Basrah, Iraq, in 1996 and 2000, respectively. She is currently an assistant lecturer of control and automation, Southern Technical University, Engineering Technical College Basrah, Iraq. She can be contacted at email: may.mohammed@stu.edu.iq

Afrah Abood Abdul Kadhim received her BSc. and MSc. in Power and Machine from the Department of Electrical Engineering, University of Basrah, Iraq in 2002 and 2006, respectively. Currently, she is an assistant lecturer at Basrah Engineering Technical College, Southern Technical University, Iraq. Her areas of interest include Power, Machine, Control, and Artificial Intelligence. 